\title{
Translating Cardiovascular Knowledge: A Global Health Perspective
}

\author{
Cristina Rabadán-Diehl • Daniel P. Judge
}

Received: 23 December 2014 / Accepted: 26 December 2014 / Published online: 13 January 2015

(C) Springer Science+Business Media New York 2015

Depending on whom you talk to, translational science may have different meanings. The scientific community agrees that moving laboratory science into preclinical settings ( $\mathrm{T} 1$ translation) and from there into humans (T2 translation) is part of the translational continuum. But beyond that, and when it comes to larger groups of people or even populations, it becomes more nebulous, and many put T3 (from patients to clinical practices) and $\mathrm{T} 4$ translation (from clinical practices to real world settings), as well as implementation science, in the same basket [1]. Yet, these are critical components of translational science.

For the last decade, more emphasis has been put into translating the knowledge that we have gained through decades of basic and clinical science research into populations. Scientists and clinicians struggle with issues like adherence to medications and promoting behavioral changes that could stop the epidemic of chronic noncommunicable diseases (NCDs). Those are complex issues as they depend not only on individuals but also on communities, health-care systems, and country policies. Developing interventions that work requires resources, and many developed countries have embarked in efforts that support research and programs, in particular, at the community level. Although the challenge is still there, we have seen some progress on childhood obesity, tobacco consumption, and early cancer detection. When it comes to

Editor-in-Chief Jennifer L. Hall oversaw the review of this article

C. Rabadán-Diehl $(\bowtie)$

Office of Global Affairs, US Department of Health and Human

Services, Washington, DC, USA

e-mail: cristina.rabadan-diehl@hhs.gov

D. P. Judge

Division of Cardiology, Johns Hopkins University, Baltimore, MD, USA

e-mail: djudge@jhmi.edu low- and middle-income countries (LMICs), the conversation, although similar, is more complex, and it has to take into account the realities of those countries, the limited resources and research infrastructures, and the triple, and sometimes quadruple, burden of disease of many of those countries.

For cardiovascular disease (CVD), and since the identification of the major risk factors through the Framingham heart study, we have been able to decrease mortality significantly. In the USA, for example, the mortality due to CVD has decreased by $50 \%$ in a few decades due in part to better prevention, control, and treatment [2]. However, CVD continues being the number one killer in the USA. Part is due to still inadequate detection and management of individuals at high risk and the adoption of unhealthy behaviors (poor diet, physical inactivity, tobacco use, and alcohol consumption), but lack of access to care and chronic disease management play a critical role. Sixty-three percent of the global mortality is due to NCDs, with CVD being the leading cause of NCD death (48\%). About $80 \%$ of NCD-related deaths are ocurring in LMICs, as indicated by WHO (http://www.who.int/chp/ncd global_status_report/en). In 2010, the National Heart Lung and Blood Institute commissioned the Institute of Medicine a report on cardiovascular health in the developing world (www.iom.edu/globalcvd). Five years later, we have made some progress (http://www.scientificamerican.com/products/ cardiovascular-health), but a lot needs to be done. The areas of diagnostics and technology present tremendous promise, but it is crucial that before interventions are scaled up, evidence-based and rigorous scientific approaches are utilized to demonstrate their effectiveness.

In recent issues of the Journal of Cardiovascular Translational Research, we presented four articles intended to review the current state of the science and provide opportunities for research and infrastructure building. We started with a focus on mobile technology, which has revolutionized the world, 
and for the last few years, it has made its way into the health field. But we need to be careful and not rush. Peiris and colleagues, with their comprehensive review of the literature to determine the impact of mHealth interventions for NCDs, have found that very few studies were high caliber and, very importantly, most lack a careful analysis of the impact of those interventions on health-care quality [3].

Identification of subclinical CVD has shown to be of great value for the early detection and prevention of cardiovascular events and is being used frequently in high-income countries, particularly on high-risk patients. Imaging technologies are currently widely used, but translating those technologies into LMICs settings poses many challenges like cost and lack of adequate clinical infrastructure. However, there are imaging modalities that are suited for implementation in LMICS, as presented by Vedanthan et al. in their excellent review [4]. The authors highlight the importance of understanding the challenges, the potential gain as well as the need to conduct studies that will focus on the feasibility and cost-effectiveness of those applications in low resource settings.

Access to and use of appropriate medical devices in LMICs have been part of a dialog encouraged by WHO through their global forums, development of medical device policies, and compendiums of innovative health technologies (http://www. who.int/entity/medical_devices/en). McGuire and Weigl discuss the approaches that are needed when considering the design and testing of medical devices in populations that live in low resource settings and in particular, the considerations to keep in mind when adapting devices developed in highincome countries [5].

Advances in cardiac surgery and the application of technology to surgery have revolutionized the field of cardiology and contributed to the decrease in mortality. However, the application to cardiac surgery technology in LMICs is challenging, and for many, this remains a dream. Yacoub et al. provide a compelling argument for the need to focus on cardiac surgery and describe a transformational approach in Egypt [6]. This case study aims to illustrate potential strategies that would help LMICs to build other centers for cardiovascular surgery.

Regardless of how we name it, this component of translational science is an area that requires multidisciplinary approaches and multi-sectorial solutions. As scientists, we have the responsibility to be knowledge brokers and to advocate for discoveries to reach the end of the translational continuum, to improve the health of populations, especially those that are the most vulnerable. The area of implementation science is giving us an opportunity to connect the dots.

\section{References}

1. Drolet, B. C., \& Lorenzi, M. N. (2011). Translational research: understanding the continuum from bench to bedside. Translational Research, 157(1), 1-5.

2. Ford, E. S., et al. (2007). Explaining the decrease in U.S. deaths from coronary disease: 1980-2000. New England Journal of Medicine, 356, 2388-2398.

3. Peiris, D., Praveen, D., Johnson, C., \& Mogulluri, K. (2014). Use of mHealth systems and tools for non-communicable diseases in lowand middle-income countries: a systematic review. Journal of Cardiovascular Translational Research, 7, 677-91.

4. Vedanthan, R., Choi, B. G., Baber, U., Narula, J., \& Fuster, V. (2014). Bioimaging and subclinical cardiovascular disease in low- and middleincome countries. Journal of Cardiovascular Translational Research, 7, 701-10.

5. McGuire, H., \& Weigl, B. H. (2014). Medical devices and diagnostics for cardiovascular diseases in low-resource settings. Journal of Cardiovascular Translational Research, 7, 737-48.

6. Yacoub, M., ElGuindy, A., Afifi, A., Yacoub, L., \& Wright, G. (2014). Taking cardiac surgery to the people. Journal of Cardiovascular Translational Research, 7, 797-802. 